\title{
Il y a une éternité, il y a un siècle, il y a un an... A quoi sert le PS dans les discours de vœux?
}

\author{
Emmanuelle Labeau \\ Aston University \\ e.labeau@aston.ac.uk
}

\section{Introduction}

La présentation des vœux constitue une tradition bien ancrée dans les sociétés occidentales: les présidents français y sacrifient le 31 décembre, les monarques belge et britannique s'expriment respectivement la veille et le jour de Noël. L'une des rares incursions d'un discours politique en intégralité dans le quotidien du citoyen, le discours de vœux s'adresse à un large public grâce à son passage à la télévision à une heure de grande écoute. Cette diffusion maximale se double d'une inévitable simplification langagière, au moins au niveau lexicologique, qui fait passer le message du discours écrit et lu au discours parlé (Ockrent, 1988:161). Au niveau thématique, le rituel comprend, outre les souhaits de rigueur et des perspectives pour l'an neuf, un bilan de l'année qui se termine. Les impératifs de simplicité linguistique, tout comme l'évocation d'un passé proche et non entièrement écoulé devrait disqualifier l'emploi du passé simple (PS), passé coupé du présent et stylistiquement marqué. Toutefois, des occurrences de PS apparaissent dans les discours de vœux des présidents de la Cinquième République et du roi des Belges ; leur analyse fera l'objet de cette étude. On débutera par un rappel du statut du PS à l'oral. Après une discussion du genre «discours de vœux » et une présentation du corpus considérés, on discutera les formes du PS relevées et on tentera d'interpréter leur rôle dans le discours de voeux.

\section{L'été indien du PS à l'oral}

En français contemporain, le PS ne fait pas (ou plus ?) partie de la conversation courante. Il est bien entendu difficile d'évaluer les pratiques pour les périodes antérieures à la fin du dix-neuvième siècle pour lesquelles nous ne disposons pas de données orales. En effet, le gramophone ne fut inventé qu'en 1877-8 et seuls des enregistrements formels étaient possibles. L'introduction de l'enregistreur en 1898 facilita le recueil des données mais ce n'est que dans les années 1960 que les enregistreurs portables ont rendu possible la fixation du parler informel:

The precise dating of [the loss of the PS in the spoken norm] must elude us, however, for our earlier evidence is mainly from the written language, which retains the passé simple to this day. (Posner 1994:80-1)

Certains indices d'utilisation orale du PS dans les états anciens de la langue avaient cependant été relevés: ainsi, le PS apparaît dans les dialogues de La vie de St Alexis au onzième siècle (Yvon 1960) et de l'Heptaméron au seizième siècle:

Le passé défini [PS] mais aussi le subjonctif imparfait (plus-que-parfait) sont employés non seulement au cours des récits mais dans les conversations commentaires qui les accompagnent sur les lèvres des différents conteurs. (Camproux ${ }^{1}$, in Zezula 1969:336, note 1)

Foulet (1920:291) rapporte que les grammairiens du seizième siècle - Dubois (1531), Meigret (1550), Mathieu (1550) et Estienne (1582) - confirment tous que le PS était d'emploi courant dans le parler de Paris. Martin (1971:399) avançait que «tout porte à croire qu[e le PS] était encore vivant en français classique » mais, à partir de la fin du dix-huitième siècle, les historiens de la langue suggèrent une régression du PS dans la langue parlée, comme le prouve une étude de correspondance familière, supposée refléter l'usage oral des scripteurs, couvrant la période du dix-septième au dix-neuvième siècle (Liu 2003). Liu s'intéresse à la corrélation entre hier et le PS et le PC; la combinaison PS + hier 
constitue $98,4 \%$ des occurrences chez les auteurs du $17^{\text {ème }}$ siècle $^{2}, 48,5 \%$ au $18^{\text {ème }}$ siècle alors qu'au $19^{\text {ème }}$ siècle, la tendance est complètement inversée avec seulement $2,2 \%$.

Gadet (1999:608) indique que la période du français moderne a vu la «raréfaction orale » du PS, «amorcée depuis la fin du $18^{\text {ème }}$ siècle, très rapide au $20^{\text {ème }}$ siècle ». Toutefois, comme le notaient justement Damourette et Pichon, le manque de documents sur «la conversation courante des siècles passés » ne nous permet que des hypothèses.

Selon Huchon (2002:257), le PS s'est mieux conservé jusqu'au milieu du $20^{\text {ème }}$ siècle dans le français méridional, bénéficiant de l'existence d'une forme correspondante en occitan. Zezula (1969:338) indique que le PS continue à être utilisé «dans quelques dialectes du Midi et de la région lyonnaise ». On a affirmé dès la période moderne que le PS à l'oral était une marque de régionalisme méridional:

\begin{abstract}
L'Atlas linguistique de MM. Gilliéron et Edmont montre que, dans tout le français proprement dit, le prétérit simple [PS] est une forme morte ; un coup d'œil jeté sur les cartes 97, 338, 360, 976, 1154 suffit à l'indiquer immédiatement. Au nord de Paris, pas de trace de «passé défini »; au Sud, il faut aller jusqu'aux départements de l'Allier, de la Creuse et de la Vienne pour commencer à en trouver quelques-uns sur les cartes ; à l'Ouest, la limite est plus proche: le prétérit simple existe encore en Normandie ; de là vient peut-être que Guy de Maupassant a beaucoup employé cette forme grammaticale. Mais, là même où le prétérit simple subsiste encore, la forme composée semble dominer déjà, ou du moins fait concurrence à la forme simple. (Meillet 1948:150)
\end{abstract}

Les linguistes ont tenté d'expliquer le rejet du PS en oral contemporain pour des raisons morphologiques, euphoniques et syntaxiques. Martin (1971:399) invoque la complexité morphologique du PS: (1) le thème vocalique manque de régularité, (2) certains verbes sont défectifs (ex. clore, absoudre, dissoudre, traire, braire ou paître) et (3) les formes se confondent avec le PRES pour la plupart des verbes en $-i r^{3}$. Phonétiquement parlant, le PS se confond, à la $3^{\text {ème }}$ personne du singulier, avec le subjonctif imparfait et se rapproche, à la première personne des verbes en -er, des verbes à l'IMP (-ai / -ais). D'autres linguistes ont souligné le caractère cacophonique des $1^{\text {ère }}$ et $2^{\text {ème }}$ personnes du pluriel:

C'est que le passé simple a des consonances qui déplaisent à une oreille française, principalement à la première et à la deuxième personne du pluriel; on évite de les faire entendre et, pour cette raison, ce temps a été éliminé de la conversation. Par contre, il s'est maintenu victorieusement dans la langue écrite. (Martin-Deydé 1935:25, in Engel 1990:19)

Wilmet (1998:368-9), tout en constatant l'érosion des positions du PS en français contemporain, voit dans le ridicule attaché à l'emploi oral de cette forme la conséquence et non la cause de sa raréfaction. En plus, le PC prétendument euphonique est source fréquente de hiatus ${ }^{4}$, ce qui pourrait favoriser le maintien du PS (ex. Fut plutôt que a été).

Pour ce qui est des facteurs syntaxiques, la tendance générale qu'ont les formes d'accompli à glisser vers les emplois d'antériorité réduit les distinctions entre PC et PS et les rendrait trop subtiles (Martin 1971:401) pour l'oral. De plus, il existe de fortes affinités entre la langue parlée où « seul compte le lien avec le moment actuel » et le PC qui permet de saisir un fait passé dans son rapport à l'actualité

Généralement exclu de l'oral, le PS s'y retrouve pourtant dans des contextes formels où il serait préféré au PC (Engel 1990:19). Le PS se double alors des «connotations livresques de la langue écrite, y compris dans l'usage moins spontané des exposés (tournures figées: $i l$ s'en fut $=$ «il s'en alla », s'il en fut $=($ "s'il y en a eu », il fut un temps » (Wilmet 1998:369). Zezula (1969: 338) avance pareillement que le PS apparaît «assez fréquemment» à la radio dans les nouvelles politiques et culturelles, dans les reportages, les commentaires politiques.

Attesté dans l'oral soutenu et l'exposé, le PS pourrait aussi se retrouver dans le discours politique:

[...] si l'écrit ne présente probablement pas le média de prédilection, en amont du message, c'est bien par l'écriture que les présidents préparent, construisent et améliorent leurs discours de vœux: les témoignages concordent sur ce point. Aussi, 
Hybride d'oral et d'écrit, vecteur de solennité aussi bien que d'intimisme et d'accessibilité, le discours politique présente autant de caractéristiques propices à l'emploi du PS que de bonnes raisons de l'éviter. Une étude sur corpus s'impose pour trancher entre nos intuitions contradictoires mais, avant de nous y consacrer, penchons de plus près sur le discours de vœux.

\section{Le discours de vœux: paroles, paroles, paroles...}

Le discours politique s'adresse «au plus grand nombre pour le faire adhérer à des valeurs communes » (Charaudeau, 2005:187). Plus que tout autre, le discours de vœux correspond à cette définition. En effet, à la différence de la plupart des discours, les voeux du chef de l'état sont largement et intégralement médiatisés et pénètrent, particulièrement depuis leur télédiffusion avant le journal du soir, la majorité des foyers. Bien que Giscard affirmât en 1980 que c'était « un de ces instants - bien rares en vérité - où je peux m'adresser à vous sans être tenu par un sujet particulier », la présentation des vœux n'est pas aléatoire. Selon Finiss-Boursin (1992: 21ss), ce type de discours comprend trois parties attendues. Les souhaits de bonne année sont bien entendu incontournables et se retrouvent soit au début soit à la fin du discours, ou aux deux extrémités, rarement au milieu. La majeure partie des discours se compose d'un exposé de politique générale qui s'ouvre sur un bilan et se poursuit sur des perspectives. La rétrospective prend des formes diverses pour les présidents selon l'avancement de leur mandat: l'héritage du prédécesseur colore les discours en début de mandat; dans les années suivantes, la valorisation du bilan personnel prime si possible et les échecs sont autant que possible occultés ou minimisés comme le montrent les discours de cohabitation de Mitterrand. Les discours du roi des Belges sont moins soumis aux échéances électorales, mais répondent aujourd'hui à des pressions similaires vu les problèmes communautaires toujours croissants qui ont affaibli le pouvoir royal. Enfin, dans de rares cas, apparait un « ingrédient supplémentaire » (Finiss-Boursin 1992:22) comme lorsqu'Anémone Giscard d'Estaing se joint à son mari le 31 décembre 1975.

Passons maintenant à la description du corpus utilisé pour cette étude.

\section{Présentation du corpus}

L'étude se base sur 50 discours de vœux des présidents de la cinquième république: 11 discours du général De Gaulle, 5 discours de Georges Pompidou, 7 discours de Valéry Giscard d'Estaing, 14 discours de François Mitterrand, 12 discours de Jacques Chirac et le seul discours prononcé jusqu'ici par Sarkozy. Pour les allocutions prononcées entre 1958 et 1987, les transcriptions fournies par Finiss-Boursin (1992) ont été utilisées; les discours de Chirac et de Sarkozy proviennent des archives en ligne de l'Élysée; quant aux discours du second septennat de Mitterrand, ils proviennent du site de la Fondation Mitterrand et de L'anthologie sonore des discours de François Mitterrand. On examinera aussi en parallèle dix discours de vœux prononcés par le roi Albert II de Belgique depuis 1997 et disponible en ligne sur le site de la monarchie belge.

Les discours de présentation des vœux forment un corpus de textes homogènes dans leur périodicité, leur but et leur contexte de production. Cette comparabilité est cependant limitée par des facteurs externes et internes à l'orateur. Ainsi, d'une part, l'évolution technologique a progressivement favorisé le vecteur télévisuel; le calendrier politique (comme la proximité des élections) affecte les thèmes abordés ; l'actualité (par exemple le tsunami de 2004, l'affaire Dutroux en 1997) influence les points couverts. D'autre part, les vœux offrent aux dirigeants l'occasion de projeter une image, voire de corriger les perceptions qu'a d'eux le public. Ainsi, Giscard tentait de dissiper sa réputation de hauteur et de froideur en mettant l'accent sur la simplicité et la solidarité ; De Gaulle et Mitterrand, plus âgés, jouaient volontiers les père et «tonton » de la patrie... Néanmoins, malgré leurs différences, les discours de vœux constituent un sous-genre homogène du discours politique dans lequel nous allons maintenant rechercher les formes et les fonctions du PS. 


\section{Le PS dans les discours de vœux}

On a vu que le discours de vœux avait trois fonctions principales: la présentation des souhaits, l'ouverture sur l'an neuf, et la rétrospective sur l'année finissante. On s'attendrait donc à y trouver des subjonctifs, des futurs et des formes de passé proche. Le PS, dont la description théorique implique la coupure par rapport à l'actualité, semble peu approprié au genre. Toutefois, des occurrences - certes limitées - de PS apparaissent dans le corpus. Nous allons dans un premier temps en donner un aperçu quantitatif avant de tenter d'interpréter la « valeur ajoutée » qu'apporte l'incongru PS au discours de vœux.

\subsection{Emploi quantitatif}

Le relevé des formes montre que le PS est marginal dans le corpus: il n'apparaît que dans 15 discours ${ }^{6}$ ( $25 \%$ du corpus) prononcés par De Gaulle (6), Mitterrand, (1), Chirac (3), Sarkozy (1) et Albert II (4).

\begin{tabular}{|c|c|c|c|c|}
\hline De Gaulle & Mitterrand & Chirac & Sarkozy & Albert II \\
\hline $\begin{array}{l}\text { Fut marquée... } \\
\text { affirmée } \\
\text { répétée... précisée } \\
(1958) \\
\text { Fut }(2 x)(1960) \\
\text { Eut - s'imposèrent } \\
\text { - se succédèrent - } \\
\text { entraînèrent - } \\
\text { furent prises (1961) } \\
\text { commença - fut - } \\
\text { passa (1962) } \\
\text { Furent - fìmes - } \\
\text { détermina }{ }^{7}(1963) \\
\text { fut (1965) }\end{array}$ & Fut (1991) & $\begin{array}{l}\text { Fut (2000) } \\
\text { Fut (2) (2002) } \\
\text { Fut marquée } \\
(2003)\end{array}$ & Fut (2007) & $\begin{array}{l}\text { Fut - firent (1997) } \\
\text { furent (2002) } \\
\text { furent éprouvées } \\
(2004) \\
\text { furent - fut (2) - ó } \\
\text { fut organisé } \\
(2005)\end{array}$ \\
\hline
\end{tabular}

Le relevé quantitatif permet de formuler quelques remarques:

Des fluctuations historiques sont envisageables: ainsi, les données semblent indiquer une éclipse du PS du milieu des années 60 au début des années 90, suivie d'une réapparition quasi exclusive de la conjugaison de être. Une distribution comparable apparaissait dans les comptes rendus des finales de la Coupe du Monde de football depuis 1950 (Labeau 2007) et suggérerait que les variations diachroniques dans l'emploi des temps proviennent de l'évolution des médias (développement de la télévision comme vecteur premier d'information et passage du journal écrit du rôle d'informateur à celui de commentateur au cours de la période considérée. On pourrait ajouter que depuis le milieu des années 90, la popularité exponentielle de l'internet nous a fait repasser d'une société de l'audiovisuel à une société de l'écrit qui permettrait une re-familiarisation avec le PS.

- L'inventaire des formes montre un usage essentiellement formulaire du PS avec 14 formes de être, 8 passifs (à nouveau la conjugaison de être) et 2 occurrences de faire; seul, De Gaulle utilise le PS avec les autres verbes. La fréquence de être au PS est bien attestée :

[...] certain common irregular verbs do seem to be favoured in the PS (especially être) (Engel 1990:81)

Cette primauté (environ 20\% des formes au PS) est confirmée par nos études de différents genres : le compte rendu sportif (Labeau 2004, Labeau et Vetters 2006, Labeau 2007) et la nécrologie (Labeau, à 
paraître 2009). L'analyse de corpus synchroniques composés de discours prononcés par des contemporains aux chefs d'état ici considérés permettrait de voir si on a affaire à une réduction diachronique de l'éventail des formes, ou à des caractéristiques linguistiques personnelles.

- L'existence d'idiolectes n'est en effet pas à négliger. Finiss-Boursin (1992) souligne des facteurs personnels comme la formation classique de De Gaulle (p.24) et son idiolecte particulier (p. 34) ou le rôle de l'âge (p.24) tous susceptibles de favoriser l'emploi de formes de registre élevé comme le PS. Il faut toutefois prendre ses explications avec circonspection. Ainsi, on aurait pu s'attendre à des PS chez Pompidou, un normalien agrégé de lettres classiques (qui écrivit d'ailleurs des discours pour De Gaulle); pareillement, un autre corpus que nous avons constitué, celui des discours d'investiture, ne contient de PS que chez De Gaulle... et Nicolas Sarkozy, pourtant 'jeune' président.

- Le PS apparaît enfin quasi exclusivement à la $3^{\text {ème }}$ personne. L'affinité du PS avec les troisièmes personnes est caractéristique: elle constituait un des piliers des descriptions textuelles du système verbal de Benveniste et de Weinrich, et elle est confirmée dans le domaine de la presse écrite (Engel 1990 :7781). Le discours de 1963 offre la seule exception dans une instance de $1^{\text {ère }}$ personne du pluriel:

$$
\left(\text { Ex.13 }{ }^{8}\right) \text { Naguère, quand en fîmes-nous autant ? (DG 1963, §2) }
$$

Albert II évite soigneusement cette première personne en 2006 au prix d'une combinaison boiteuse du PS et du passé composé:

(Ex.29) Un concert de jazz fut organisé au Sablon où nous avons pu également rencontrer les responsables d'organisations qui s'investissent dans des aspects du bien commun tels que la défense de l'environnement, la création d'emplois, la formation des jeunes par l'enseignement, le sport et la culture. (A 2005)

Les formes de la deuxième personne, absentes de notre corpus, sont peu attestées et stylistiquement marquées comme solennelles ou humoristiques (Pires 2003).

\subsection{Emploi qualitatif}

Une approche purement quantitative des formes du PS n'aurait qu'un intérêt limité et nous allons donc nous interroger sur leur contexte d'emploi, notamment la position dans le discours et les thèmes pour lesquels les PS sont utilisés.

Penchons-nous d'abord sur la position des formes du PS dans le schéma du discours de vœux. Deux tendances apparaissent. La majorité des PS apparaissent soit en début de discours (Ex. 1, 2, 4-5, 9-11, 17 , 18-9, 20, 21, 26, 27-8, 29), soit en position isolée au cœur du texte (Ex. 3, 8, 12,14, 15, 16, 22, 23, 24, 25). Les emplois initiaux - isolés ou en courte série - apparaissent plutôt en propositions indépendantes alors le deuxième type d'emploi est principalement constitué d'occurrences de être en proposition subordonnée. Le PS ne remplit donc aucunement le rôle de vecteur de l'histoire qui lui est traditionnellement attribué.

Interrogeons-nous ensuite sur le cotexte d'apparition du PS. Dans notre corpus, il se combine avec des indications temporelles comme des dates : entre janvier 1947 et mai 1958 (Ex.6) ou la soirée du 21 juillet (Ex. 26), ou des adverbes tels que naguère (Ex. 13) et jadis (Ex.10) qui renforcent la coupure de l'actualité. Plus curieusement, de discours d'Albert II en 2002 combine le PS avec un déjà, une combinaison en principe 'impossible' compte tenu des informations aspectuelles contradictoires que véhiculent le temps verbal et l'adverbe:

(Ex.24) Dans le souci de promouvoir la créativité, le Palais de Bruxelles accueille depuis fin octobre des œuvres d'artistes belges contemporains. Ces œuvres furent déjà accessibles au grand public et le seront encore durant l'été prochain (A 2002)

Il faut peut-être y voir un désir d'éviter le double hiatus qu'aurait provoqué l'emploi du passé composé ('ces ouvres ont déjà_été_accessibles'). 
Voyons maintenant s'il existe un lien entre les thèmes abordés et l'utilisation du PS. Dans les discours d'Albert II, le PS marque les événements marquants de l'année écoulée: la marche blanche contre les rapts d'enfants en 1997 (Ex.22, 23), la catastrophe du zoning industriel de Ghislenghien en 2004 (Ex. 25) ou les points forts des festivités pour le $175^{\text {ème }}$ anniversaire de la Belgique en 2005. (Ex.26-29). On trouve aussi des PS pour récapituler l'année écoulée: c'est le cas de toutes les instances relevées chez Chirac (Ex.17-20) et chez Sarkozy (Ex.21). C'est également le cas dans les discours de De Gaulle en 1958 (Ex.1) et dans une certaine mesure en 1962 (Ex.9-11). Dans ce même discours (Ex.12, 13), le PS joue aussi son rôle majeur dans les discours du Général, à savoir le marquage du passé négatif auquel sa présidence a mis fin (voir aussi Ex. 4-7, 14). Il est à noter que cet emploi de passé coupé de l'actualité correspond au profil traditionnel de la forme.

Une dérive sémantique pourrait donc s'être produite: le PS, utilisé initialement pour référer à des événements clairement coupés de l'actualité de l'orateur, s'est progressivement mué en élément de mise en relief d'un événement marquant. On est donc passé d'un marqueur de distanciation à un marqueur de « dimensionalisation », pour reprendre la taxinomie de Monville-Burston \& Waugh (1985).

\section{Conclusion}

Que permet de conclure cette brève analyse des discours de vœux du dernier demi-siècle ?

D'abord, la présence du PS dans ce genre le distingue de l'oral spontané et trahit l'origine écrite du discours. Pourtant, cet emploi semble être devenu formulaire si l'on en croit le recours fréquent aux formes de la $3^{\text {ème }}$ personne d'être. Il est difficile d'identifier les causes de l'évolution perçue dans ce corpus. Une explication possible serait l'évolution de la perception du chef de l'état: De Gaulle, «le plus grand des Français », utilisait des formes solennelles qui passeraient mal aujourd'hui de la part de présidents qui s'affichent à la une de la presse people. Une autre option serait la modification du paysage médiatique: la pénétration de la télévision dans l'énorme majorité des foyers a fait passer le discours de vœux d'un texte lu à un texte joué où l'image a pris une importance majeure ${ }^{9}$ et le verbe s'est simplifié pour retenir l'attention du téléspectateur toujours prêt à zapper.

La raréfaction du PS semble s'être doublée d'une recharge sémantique de la forme dont la présence souligne non plus la distance temporelle mais l'importance ou la solennité des événements rapportés.

Le corpus étudié ici est bien entendu trop limité pour permettre des généralisations, il faudrait avoir accès à un éventail plus large de discours. Ainsi, le discours de vœux est plus tourné vers l'avenir que le passé et des discours de commémoration risqueraient de contenir plus de PS. Le recours au PS pourrait trahir un idiolecte et l'examen d'un éventail plus large de discours pour chaque chef d'état permettrait d'identifier d'éventuels tics et préférences linguistiques. L'hypothèse du regain du PS dans les années 1990 mériterait aussi d'être vérifiée par l'étude de discours politiques par époque plutôt que par personne. Bref, on est loin d'avoir fait le tour de la question!

\section{Références}

Brunot, F. (1966). Histoire de la langue française des origines à nos jours (tomes I, IV, 2 et VI,2). Paris : Colin.

Charaudeau, P. (2005). Le discours politique: Les masques du pouvoir. Paris: Vuibert.

Engel, D.M. (1990). Tense and text: A study of French past tenses. Routledge: London, New York.

Finiss-Boursin, F. (1992). Les discours de voux des présidents de la République: La France au fond des yeux. Paris: Librairie générale de droit et de jurisprudence.

Foulet, L. (1920). 'La disparition du prétérit' Romania 46 :271-313.

Gadet, F. (1999) 'La langue française au XXe siècle : L'émergence de l'oral', in Chaurand, J. (dir.) Nouvelle histoire de la langue française. Paris, Seuil, p.583-671. 
Huchon, M. (2002). Histoire de la langue française. Paris: Le livre de Poche no.542.

Labeau, E. (2004). 'Le(s) temps du compte rendu sportif francophone', Journal of French Language Studies 14, pp.129-148.

Labeau, E. (2007). 'Et un, ou deux, ou trois ? Les temps-champions du compte rendu sportif depuis 1950', in Labeau, E., Vetters, C. \& Caudal, P. (eds). Sémantique et Diachronie du système verbal français. Amsterdam / New York, Rodopi, pp.203-233.

Labeau, E. (forthcoming 2009).'Le PS, cher disparu de la rubrique nécrologique ?’ Journal of French Language Studies, , 19/1.

Labeau, E. \& Vetters, C. (2006). 'Le PS est-il toujours en course ?', communication orale à la conférence Chronos 7, Anvers, septembre 2006.

Liu, Y.-C. (2003). 'Le passé simple et le passé composé: leur concurrence dans l'histoire du français'. Présentation à l'atelier Diachronie et sémantique du système verbal français. Birmingham, Aston University, 11-12 septembre 2003. (communication personnelle)

Martin, R. (1971). Temps et Aspect: Essai sur l'emploi des temps narratifs en moyen français. Paris: Klincksieck.

Martin-Deydé, G. (1935). Comment employer les modes et les temps des verbes en français. Paris : F. Nathan.

Meillet, A (1948). 'Sur la disparition des formes simples du prétérit' in Linguistique historique et linguistique générale. Paris: Honoré Champion, pp.149-158.

Monville-Burston, M. \& Waugh, L. (1985). 'Le passé simple dans le discours journalistique', Lingua 67:121-170.

Ockrent, C. (1988). Duel. Comment la television façonne un Président. Paris: Hachette.

Pires, M. (2003) 'Les deuxièmes personnes du passé simple aujourd'hui', communication orale au colloque annuel de l'Association for French Language Studies (AFLS), Tours, 26 septembre 2003).

Posner, R. (1994) 'Historical linguistics, language change and the history of French', Journal for French Language Studies4:75-97.

Yvon, H. (1960) 'Emploi dans la Vie de Saint Alexis (11 ${ }^{\text {ème }}$ siècle) de l'imparfait, du passé simple et du passé composé de l'indicatif', Romania 81:244-250.

Wilmet M. (1998). Grammaire critique du français, Paris / Bruxelles: Hachette supérieur / Duculot. (2e éd.)

Zezula, J. (1969). 'Le passé simple dans la langue de la presse d'aujourd'hui', Beiträgue zur Romanischen Philologie 2:69:336-345.

Anthologie sonore des discours de François Mitterrand (1981-1995). La Librairie sonore.

http://www.monarchie.be

http://www.élysée.fr

\section{Annexes}

(1) Votre décision fut marquée lors de la crise nationale du mois de mai, affirmée par le référendum, répétée par les élections, précisée par le vote des élus dimanche dernier. (De Gaulle 1958, §1, phrase 2) 
(2) Solide, laborieuse, cohérente, je crois que jamais, en dépit des difficultés, [la France] ne fut plus capable de saisir ses propres chances et d'être utile aux hommes et à la paix (De Gaulle 1960, §1, phrase 5)

(3) Françaises, Français, je vous le demande, donnez au projet qui vous est soumis une approbation immense. D'abord, parce que c'est le bon sens, mais aussi pour cette raison que l'enjeu dépasse de beaucoup les préférences théoriques, les intérêts particuliers, les attachements partisans et que jamais ne fut plus nécessaire une cohésion nationale. (DG 1960, §6, phrase 2)

(4-5) Il y aura bientôt quatre ans qu'ayant quitté le chemin du déclin, elle a pris la route qui monte. Cela eut lieu - qui peut l'oublier ? - en raison de certaines évidences qui s'imposèrent à la nation: impuissance du régime des partis [...] (DG, 1961, §2)

(6) Si, dans l'ordre politique, l' inconsistance était, entre janvier 1947 et mai 1958, qu'en cet espace d'environ onze ans se succédèrent vingt-deux cabinets, ensuite notre pays pendant presque quatre années a eu un seul gouvernement [...] (DG 1961, §7)

(7) $\mathrm{Si}$, dans le domaine social, on constate que pour 9 millions d'ouvriers français, les conflits de travail, sous le régime précédent, entraînèrent chaque année, en moyenne, 7 millions de journées de grève, sous le régime actuel, ce n'est plus qu'un million par an. (DG 1961, §8)

(8) Certes, les rudes mesures qui furent prises, lors de la grande opération financière, monétaire et économique que l'on sait ont été pour beaucoup dans ce changement total de la tendance. (DG 1961, §8)

(9-11) Quand commença 1962, on se tuait encore en Algérie, tandis qu'attentats et complots se prolongeaient encore en métropole. Récemment, un démon qui nous fut jadis très familier et très malfaisant, celui des crises politiques, a cru trouver l'occasion de revenir nous tenter. Enfin, il y a deux mois, le monde passa près de la guerre ((DG 1962, §2, phrases 2-4).

(12) Pour la France, l'année qui finit a été, en somme, favorable. Par contraste avec d'autres temps qui furent cruels et agités et en dépit des annonces alarmantes des partisans inassouvis, nous n'avons pas, en la saluant, à évoquer de catastrophes. (DG $1962, \S 1)$

(13) Naguère, quand en fîmes nous autant? (DG 1962, §2)

(14) Mais après une période de drame et de médiocrité qui détermina tant de gens à tenir notre patrie pour vouée à l'effacement, voici qu'on s'est repris partout à en attendre de grandes entreprises. (DG 1963, §5)

(15) Au terme d'une année qui fut celle de notre fusée Diamant, de nos premiers satellites, des succès de nos savants dans le domaine de la biologie moléculaire, du classement de notre procédé de télévision en couleurs au premier rang du concours mondial, nous sommes fondés à attendre beaucoup de nos recherches scientifiques et techniques. (DG 1965, §4)

(16) Le départ, ces derniers jours, de M. GORBATCHEV par qui tout fut possible: la fin de la guerre froide, le désarmement, l'approche de la démocratie sur cette terre brûlée par des siècles de despotisme, laisse place à des républiques souveraines à qui nous adressons nos voeux mais qui ont encore à trouver leur voie pour que RECULENT la misère et la faim, et pour qu'on SACHE où siège la responsabilité nucléaire. (M 1991)

(17) Si le passage à l'an 2000 a été célébré dans le monde entier comme le commencement d'une époque nouvelle, l'année 2000 fut, en réalité, un temps de prise de conscience. (C 2000, §2)

(18-9) 2002 fut une année de débats et, pour certains, l'occasion d'exprimer leur insatisfaction et leurs inquiétudes. Elle fut également, et elle restera, l'année du sursaut républicain. (C2002, §2) 
(20) 2003 fut marquée dans le monde par l'instabilité, les tensions et les crises: la guerre en Irak, le terrorisme, les attentats et les violences au Proche-Orient, les troubles en Côte d'Ivoire... (C 2003, §2)

(21) En cette fin d'année 2007 une première étape s'achève sur la voie du changement. Ce fut celle de l'urgence: urgence à dépasser les vieux clivages partisans. (S 2007)

(22) Nous voulons un pays où les familles immigrées SOIENT pleinement INTEGREES dans notre vie nationale, comme ce fut le cas à l'occasion de la marche blanche. (A 1997)

(23) Nous voulons que la Justice soIT plus humaine, plus efficace et que le secret de l'instruction SOIT RESPECTE. Nous voulons un pays où Flamands, Wallons et Bruxellois MARCHENT ensemble, côte à côte comme ils le firent dans la marche blanche. (A 1997)

(24) Dans le souci de promouvoir la créativité, le Palais de Bruxelles accueille depuis fin octobre des œuvres d'artistes belges contemporains. Ces œuvres furent déjà accessibles au grand public et le seront encore durant l'été prochain (A 2002)

(25) Mesdames et Messieurs,

En ce temps de Noël où les familles se retrouvent, la Reine et moi pensons spécialement à toutes celles qui furent cruellement éprouvées par la catastrophe de Ghislenghien. (A2004)

(26) Les journées populaires qui entouraient le 21 juillet furent rayonnantes de bonne humeur. (A 2005)

(27-8) Le concert donné la veille de notre fête nationale à l'initiative du Concours Musical Reine Elisabeth fut particulièrement réussi. La soirée du 21 juillet à Bruxelles, avec le spectacle de la grande péniche descendant le Boulevard Botanique fut mémorable. (A2005)

(29) Un concert de jazz fut organisé au Sablon où nous avons pu également rencontrer les responsables d'organisations qui s'investissent dans des aspects du bien commun tels que la défense de l'environnement, la création d'emplois, la formation des jeunes par l'enseignement, le sport et la culture. (A 2005)

\footnotetext{
${ }^{1}$ Camproux, C. 'Téléscopage morpho-syntaxique', Le français moderne, juillet, pp.163-4 [sic].

${ }^{2}$ Brunot (T.I:977, note 1) donne des extraits littéraires et épistolaires et mentionne que «Les exemples classiques sont innombrables ».

${ }^{3}$ Martin (1971:400, note 738) en compte environ 350.

${ }^{4}$ Wilmet (1998:367) mentionne que Racine, pour éviter le hiatus, ne craint pas d'enfreindre la règle des vingt-quatre heures: "Le ciel avec horreur voit ce monstre sauvage; / le monde s'en émeut, l'air en est infecté;/ le flot qui l'apporta recule épouvanté » (Phèdre, V, 6).

5 Martin (1971:401-2) invoque aussi l'argument guillaumien que le PC offre une vision rétrospective plus adaptée au regard porté par le locuteur sur le passé que la vision prospective du PS.

${ }^{6}$ Le discours de Mitterrand en 1987 comprend des subjonctifs imparfaits morphologiquement liés au PS: fût défendue, écoutée, respectée.

${ }^{7}$ Ce discours comprend aussi des imparfaits du subjonctif: n'eussent-été, fût, englobât, laissât.

${ }^{8}$ Ce numéro correspond au classement dans l'annexe.
} 
Sociolinguistique et écologie des langues

DOI $10.1051 / \mathrm{cmlf0} 8144$

${ }^{99}$ Finiss-Boursin (1992:43) mentionne que la première allocution télévisée de De Gaulle en 1958 avait été un fiasco, le général se contentant de sortir ses lunettes et de lire son texte, tout habitué qu'il était aux discours radiodiffusés. Conscient du pouvoir des nouveaux médias, De Gaulle avait par la suite pris des cours à la Comédie-Française. 